\title{
Performance Evaluation of Voice over IP over WiMAX
}

\author{
Zobida Abbas Mohamed ${ }^{1}$, Dr. Mohmmed Abakar ${ }^{2}$ \\ Department of Communication Engineering, Al-Neelain University
}

\begin{abstract}
Worldwide Interoperability for Microwave Access (WiMAX) in the wireless world is one of the hottest and latest technologies and its standard is based on IEEE 802.16 wirelesses Technology. It is one of the promising technologies due to its high data rate, large coverage area and deployment low cost which provide wireless last-mile connectivity. It is also a promising technology in wireless where it supports application of high bandwidth. Whereas Voice over Internet Protocol is also a promising new Technology which over the Internet Protocol based network provides the access to voice communication, this technology is an alternative of Public Switched Telephone Network(PSTN) because of incapability to transmit voice in the form of packets over the network of IP. The paper represented here, in this we analyze the different VoIP Codec performance over the network of WiMAX. The performance metrics such as packet end to end delay, MOS and jitter has been taken to evaluate the various VoIP Codec performances.
\end{abstract}

Keywords: WIMAX, VOIP, MOS.

\section{Introduction}

Multimedia Technologies are now a days Gaining much more attention of the user due to its broadband Technologies[1].In recent years device of user is changing, Firstly they were using email and net but they now need to use multimedia services such as video conferencing, streaming and VoIP etc. WiMAX which is standard based on the IEEE802.16 wireless technology is a very best wireless technology when compared with wireless LAN or 3G, which provide radio link connectivity to the last mile because of its high speed rate of data, low cost for its deployment and which cover's large coverage area. IEEE802.16 has been discovered in order that it can not only replace XDSL for the customer having small business but also as a technology in which mobile internet access is used [2]. One of the most killer applications of the 802.16 technology is VoIP (Voice over IP) which support bidirectional voice conversation. WLAN provides the infrastructure which is cost effective to its service provider and as well as promise the Quality of Service to its user without creating complexity in the network of the core whereas if we see WiMAX which is a technology that can be easily deployed and integrated with IP core network[3]. The study was made on the Quality of service parameters which had impact on the service of the WiMAX performance of the WiMAX network. The conclusion gained from the study was that these QoS parameters are the very much required to increase the WiMAX network performance. Using various. Voice codec simulations and statistical distribution was studied and the different performance parameter was evaluated to search out the best choice of VoIP codec [4]. In earlier work study was made only for the Best Effort Service flow and its analisation was done on the parameters such as end -to-end delay, MOS and jitter.

The aim of the paper here is to examine deploy the QoS over the network of the WiMAX and analyze it's capability to deliver correct QoS to the application of voice or voice application. The method follows here as: Firstly a WiMAX network using OPNET is created and then simulation is carried out for the MOS, jitter, and packet end-to-end delay. The rest of the paper is organised is as follows: Sectoin2 gives the overview of the working of the VoIP. Section 3 describes the various VoIP codec's. Section 4 present simulation result and analysis. Finally in section 5 we conclude the result.

\section{Working of the VoIP}

For the transmission of the voice in the form of packet over the networks of IP VoIP uses protocol called Internet Protocol. The process involved in the transmission of the voice over the IP involve are Firstly the analog signal of the voice is digitized and then from the digitized signal unwanted noise signal is isolated and finally the voice signal is compressed using compression algorithm or codec's. After the voice compression it is packetized and send over the network of IP, here each packet which is being transmitted needs a sequence number, a destination address and a data that need to check error. At this stage signalling protocol is attached to achieve the following requirements. At the receiver side when the packet of the voice arrives at the destination the sequence number in it helps the voice packet to place it in according to sequence and finally to recover the original data from the packets decompression algorithm is applied to it.

\section{VoIP CODEC'S}

When TCP protocols is in favour of the reliability over the timeliness then RTP and UDP are the two protocols which are the logical choice to carry voice. This means each analog voice signal is first converted into digital and then back. The analog signal is first sampled on the sampling rate of $8 \mathrm{KHz}$ which is very frequent sample used in most of the cases. Next the output of this is then encoded based on many factors, the compression, frames length or the framing line. After this the frame is finally encapsulated for the transmission through the network. The frame is encapsulated into the RTP/UDP/IP packets. All this following is carried out by one among various voice codec's. The various voice 


\section{International Journal of Science and Research (IJSR) \\ ISSN (Online): 2319-7064}

Index Copernicus Value (2013): 6.14 | Impact Factor (2015): 6.391

codec differ from each other in the encoding algorithm, coding delay, quality of required bandwidth etc [5][6][7].

G.711 is the voice codec's which is International standard for telephone audio encoding whose bit rate is fixed i.e. $64 \mathrm{kbps}$.

G.729 is also a low bit rate codec it is an industry standard with utilization of bandwidth is high for the voice call of toll quality.

G.723.1 is one the most efficient codec's with the highest compression ratio when compared with other codec's and are low bit rate codec. It is used in the application of video conferencing.

G.726 uses ADPCM Standard speech codec which transmit the data at the rate of $16,24,32,40 \mathrm{kbps}$.

G.728 voice codec using low delay code excited linear prediction codes aspect at the rate of $16 \mathrm{kbit} / \mathrm{s}$.

\section{Simulation Result and Analysis}

To evaluate the performance of the VoIP it is necessary to study the real time scenario. Here the simulation tool is used known as OPNET 14.5A which reflect the actual deployment of the WiMAX network. The two scenarios are studied here:

For static nodes. For mobile nodes

\section{A) Static Node}

Fig1 shows the simulation setup which is used for the WiMAX network. A 7 celled WiMAX network is deployed using the wireless Deployment Wizard of OPNET. In this network multiple subscriber stations is deployed in the range of Base Station. The core network and the Base Station is connected with each other by the server backbone via the IP backbone. The server backbone is connected further to the voice server configured as SIP server. The service provider company network is formed together by the combination of Base Station, IP backbone, Server backbone and the Voice server. The radius of the cell is set to $30 \mathrm{~km}$. The power of Base Station transmission is set to $10 \mathrm{~W}$ and the power of the Subscriber Station is set to $0.5 \mathrm{~W}$ based on [8]. In the cell 2 and 3 the number of subscriber is set 10and the voice o ver IP calls are set between them using SIP in mesh form.

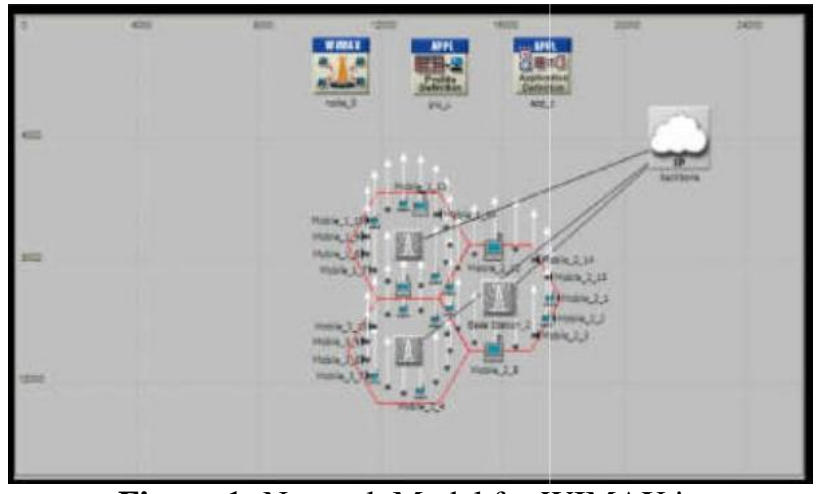

Figure 1: Network Model for WIMAX in

\section{OPNET}

\subsection{Average Jitter}

Quality of voice is best when it has the zero jitter. As shown in the Fig2 average voice jitter for the various codec's G.723.1 with $5.3 \mathrm{~kb}$ ps and $6.3 \mathrm{kbps}$ and G.726 with $32 \mathrm{kbps}$ is almo st zero which signifies a quality of voice is very $\mathrm{g}$ ood[9] whereas some deviation is shown by all other codes.

To increase the number of users the technique of silence suppression is used. Since it prevents the packetization of the silence suppression is used. Since it prevents the packetization of the silence and therefore bandwidth is saved. But on the other it increases positive jitter considerably of G.726 codec compared to the other vo ice codec. As shown in Fig3 .Hence G.726 cannot be used in case of this technique.

\subsection{Average Packet End-To-En d Delay}

Packet switching is the principle o $\mathrm{n}$ which packet network operate. Factors which affects the packet end to end delay includes network delay, Look delay, Packetization delay, Serialization Delay etc. Fig 2 shows that for the voice co dec G.723.1 the packet end to end delay is the hig hest irrespective of the silence suppression [8]. Since code rate of G.723.1 is $5.3 \mathrm{kbps}$ and $6.3 \mathrm{kbps}$ which result in the packet formation of larger count ha ving small size. As number of packet increases, congestion also increases on the network and vice versa.

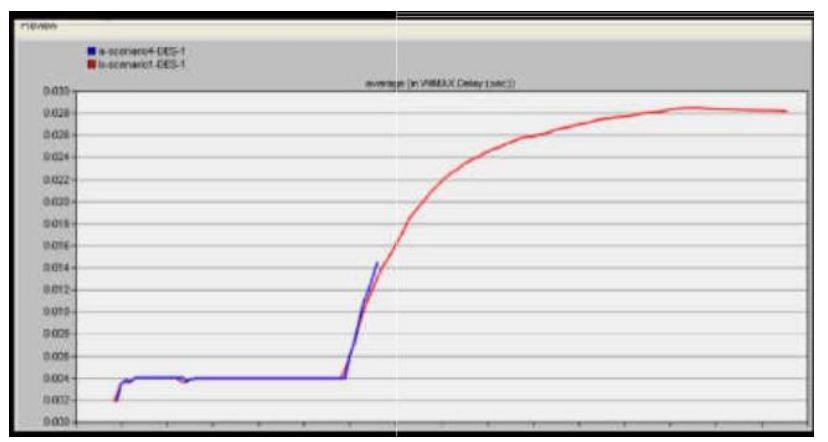

Figure 2: Average Packet End-To-End Delay

\section{A) Mobile Node}

Figure 3 shown below is the simulation set up which is used for WiMAX network .A 7-celled WiMAX network with multiple subscriber station which is in the range of Ba se Station is deployed using the wireless Deployment Wizard of OPNET. The Base Station and the core network are connected to each other by server backbone through an IP backbone and also through an ASN gateway which controls the mobile nodes mobility. The server backbone is also connected further to the voice server which is configured as the SIP server. Service Provider Company network is formed by the combination of the Base Station, IP backbone, ASN gateway, se rver backbone and the voice server. To control the mobility of the mobile nodes a Generic Routing E ncapsulation tunnel is set between the Base Station and the ASN gateway. The radius of the cell is set to be $30 \mathrm{~km}$. The mobile nodes are allowed to move at the rate of 


\section{International Journal of Science and Research (IJSR) \\ ISSN (Online): 2319-7064 \\ Index Copernicus Value (2013): 6.14 | Impact Factor (2015): 6.391}

$50 \mathrm{~km} / \mathrm{hr}, 100 \mathrm{~km} / \mathrm{hr}$ and $150 \mathrm{~km} / \mathrm{hr}$. The power of Base Station and the Subscriber Station is set to $10 \mathrm{~W}$ and $0.5 \mathrm{~W}$ based on [8] res pectively. In cell 2 the number of subscriber is 10 and in cell 3 and 7 is 5 each. Now the various voice codec's are used for voice over IP calls and the variation in the following parameters is studied i.e. jitter and packet endto-end delay. Also very few voice codec's could be modeled that are G.723.1 with 5.3kbps, G 726 with 32kbps and G 728 with $16 \mathrm{kbps}$.

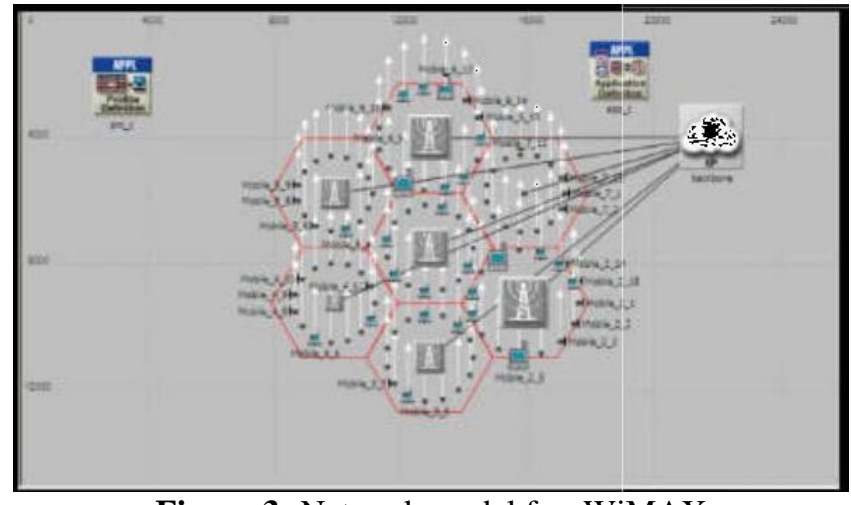

Figure 3: Network model fo r WiMAX

\section{B) Average Jitter and Packet E nd-To-End Delay:}

In the mobile scenario there is more packet loss in the physical layer and here interpret ed by the upper layer as delay this is due to the pr operty of hiding of the MAC or Link Layer Protoco 1 [10]. In G711 size of the packet is larger therefore it has higher probability of loss than G723.1 thereby has higher delayed when compared to G723.1 [10]. We can say that as the Subscriber Station is mobile in nature therefore Base Station supporting it changes frequently due to handov er phenomenon. The number of packet for $\mathrm{G} 723$. 1 is much more than G711 therefore G723.1 suffers maximum jitter as shown in Fig 5.

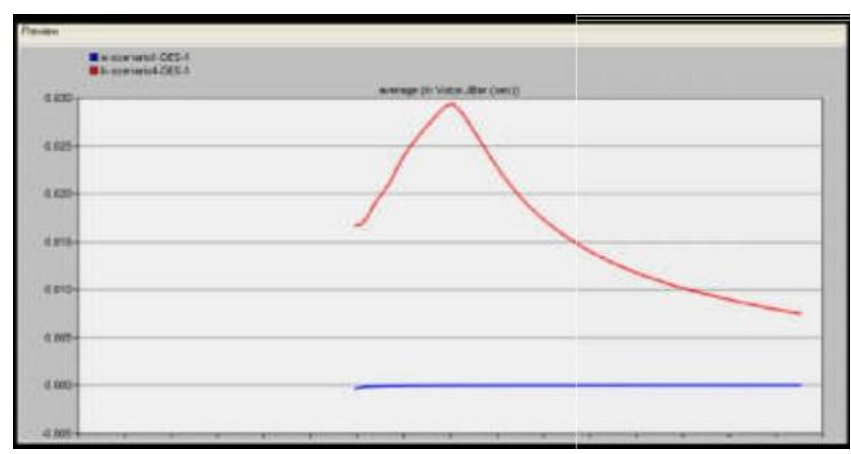

Figure 5: Mean jitter

\section{Conclusion}

The aim of this paper was to observe under various mobility scenarios the variation of packet end-to-end delay and jitter. This work here shows the variation of the following parameter with respect to stationary network in which all the nodes are kept stationary with respect to the mobile network where each node is mobile in nature and has a varying speed. The observation is made that delay is more in the case of the stationary nodes compared to the mobile nodes. The figure shows that jitter increases considerably in stationary network when in the network silence suppression is activated and it is observed that it increases more when in the network is mobile. The reason behind this is that when node becomes mobile it undergoes handover. Thus in the delivery of the packet an inconsistency is created and thereby jitter. From the figure 4 and 5 it is observed that when silence suppression is activated then in the overall network delay decreases. The reason is due to queuing delay. With the silence suppression the number of packet decreases therefore the packets number which is queued in the intermediate also decreases. But when mobility is introduced in the network then the packet end-toend delay decr eases considerably.

\section{References}

[1] N. Scalabrino, F. D. Pel egrini, I. Chlamtac, A. Ghittino, and S. Pera,"P erformance evaluation of a WiMAX testbed under VoIP traffic" in Proc. First ACM Inter national Workshop on Wireless Network Test-beds; experimental evaluation and Charact erization (WiNTECH), September 2006, pp. 97 -98.

[2] IEEE Std 802.16e-200 5, "IEEE Standard for Local and Metropolitan Area Networks - Part Air Interface for Fixed and Mobile Broadband Wireless Access Systems Amendment 2," Feb. 28,2006.

[3] Kh. Shuaib, "A Perform ance Evaluation Study of WiMAX Using Qu alNet", WCE, ICWN 2009, July 13, London, UK.

[4] S. Alshomrani, S. Qamar, S. Jan, I. Khan and I. A. Shah, "QoS of VoIP over WiMAX Access Networks",

[5] International Journal of Computer Science and Telecommunications, V ol. 3, Issue 4, April 2012.

[6] S. Karapantazis and F.-N. Pavlidou, "VoIP: A comprehensive survey on a promising technology",

[7] Computer Networks, V ol. 53, Issue 12, pp. 2050-2090, 2009.

[8] Kumar and S. Ganesh T horenoor, "Analysis of IP Network for differe nt Quality of Service", International Symposium on Computing, Communication, and Control (ISCCC 2009) Proc. of CSIT Vol.1 (2011) (C) (2011) IACSIT Press, Singapore.

[9] T. Zourzouvillys and E. Rescorla, "An introduction to standards-based VoIP: SIP, RTP, and friends", Internet Computing, IEEE, Vol. 14, Issue 2, pp. 69 -73, 2010.

[10] http://en.wikipedia.org/wiki/G723.1

[11] http://www.voip-info.org/wiki/view/ITU+G.726S.

[12] Jari Korhonen, Ye Wang, School of Computing, National University of Singapore, "Effect of Packet Size on Loss Rate and Delay in Wireless Links", IEEE Communications Society, WCNC, 2005, pp 1608-1613. 